\title{
Residential exposure to transportation noise in Denmark and incidence of dementia: national cohort study
}

\author{
Manuella Lech Cantuaria, ${ }^{1,2}$ Frans Boch Waldorff, ${ }^{3,4}$ Lene Wermuth, ${ }^{5,6}$ Ellen Raben Pedersen, ${ }^{1}$ \\ Aslak Harbo Poulsen, ${ }^{2}$ Jesse Daniel Thacher, ${ }^{2}$ Ole Raaschou-Nielsen, ${ }^{2,7}$ Matthias Ketzel, ${ }^{7,8}$ \\ Jibran Khan, ${ }^{7,9}$ Victor H Valencia, ${ }^{7}$ Jesper Hvass Schmidt, ${ }^{10,11,12,13}$ Mette Sørensen ${ }^{2,14}$
}

For numbered affiliations see end of the article

Correspondence to:

M L Cantuaria

mlca@mmmi.sdu.dk

(ORCID 0000-0003-3201-9606) Additional material is published online only. To view please visit the journal online.

Cite this as: $B M J$ 2021;374:n1954 http://dx.doi.org/10.1136/bmj.n1954

Accepted: 2 August 2021

\section{ABSTRACT}

OBJECTIVE

To investigate the association between long term residential exposure to road traffic and railway noise and risk of incident dementia.

\section{DESIGN}

Nationwide prospective register based cohort study.

SETTING

Denmark.

\section{PARTICIPANTS}

1938994 adults aged $\geq 60$ years living in Denmark between 1 January 2004 and 31 December 2017.

\section{MAIN OUTCOME MEASURES}

Incident cases of all cause dementia and dementia subtypes (Alzheimer's disease, vascular dementia, and Parkinson's disease related dementia), identified from national hospital and prescription registries.

RESULTS

The study population included 103500 participants with incident dementia, and of those, 31219 received a diagnosis of Alzheimer's disease, 8664 of vascular dementia, and 2192 of Parkinson's disease related dementia. Using Cox regression models, 10 year mean exposure to road traffic and railway noise at the most ( $\mathrm{L}_{\text {den }}$ max) and least $\left(\mathrm{L}_{\text {den }}\right.$ min) exposed façades of buildings were associated with a higher risk of all cause dementia. These associations showed a general pattern of higher hazard ratios with higher noise exposure, but with a levelling off or even small declines in risk at higher noise levels. In subtype analyses, both road traffic noise and railway noise were associated with a higher risk of Alzheimer's disease, with hazard ratios of 1.16 (95\% confidence interval 1.11 to 1.22 ) for road $L_{\text {den }} \max \geq 65 \mathrm{~dB}$

\section{WHAT IS ALREADY KNOWN ON THIS TOPIC}

Environmental exposures have received growing attention for possible roles in the development and pathogenesis of dementia

Previous research on transportation noise and dementia is scarce and diverse The few existing studies on the topic generally indicated no association between transportation noise and dementia, but some suggested an association with cognitive impairment in adults

\section{WHAT THIS STUDY ADDS}

Long term residential exposure to road traffic and railway noise at the most and least exposed façades of buildings was associated with increased risk of all cause dementia and dementia subtypes, especially Alzheimer's disease

The associations between road traffic and all cause dementia followed a positive exposure-response association, with a levelling off or small declines in risk at

high noise levels compared with $<45 \mathrm{~dB}, 1.27$ (1.22 to 1.34$)$ for road $\mathrm{L}_{\mathrm{den}} \min \geq 55 \mathrm{~dB}$ compared with $<40 \mathrm{~dB}, 1.16$ (1.10 to 1.23) for railway $L_{d e n} \max \geq 60 \mathrm{~dB}$ compared with $<40 \mathrm{~dB}$, and 1.24 (1.17 to 1.30 ) for railway $L_{\text {den }}$ min $\geq 50 \mathrm{~dB}$ compared with $<40 \mathrm{~dB}$. Road traffic, but not railway, noise was associated with an increased risk of vascular dementia. Results indicated associations between road traffic $L_{\text {den }}$ min and Parkinson's disease related dementia.

\section{CONCLUSIONS}

This nationwide cohort study found transportation noise to be associated with a higher risk of all cause dementia and dementia subtypes, especially Alzheimer's disease.

\section{Introduction}

Dementia is one of the greatest health challenges of the 21st century. ${ }^{1}$ With the shifting age distribution of the global population, the number of people with dementia is expected to exceed 130 million by $2050 .^{2}$ In view of the rapid progress of this disease and the increased costs to the health and social care system, the identification of potential modifiable risk factors for dementia have become highly relevant for prevention and management. ${ }^{1}$ Besides well established risk factors, such as cardiovascular diseases and unhealthy lifestyle, environmental exposures have received growing attention for a possible role in the development and pathogenesis of dementia. ${ }^{13}$

Transportation noise is considered the second worst environmental risk factor for public health in Europe, only surpassed by air pollution. ${ }^{4}$ About $20 \%$ of the European population is exposed to transportation noise above the recommended guideline levels of 55 $\mathrm{dB},{ }^{56}$ which is equivalent to noise levels in an open office environment and is $5 \mathrm{~dB}$ lower than a regular conversation between people at a distance of $1 \mathrm{~m}$ (60 $\mathrm{dB}){ }^{7}$ Epidemiological studies have consistently linked transportation noise to various diseases and health conditions, such as coronary heart disease, obesity, and diabetes. ${ }^{68}$ The proposed biological mechanisms for an effect of noise on health are noise induced stress reactions, with activation of the autonomic nervous and the endocrine system and subsequent release of stress hormones, affecting several physiological functions. ${ }^{9}{ }^{10}$ Exposure to noise during the night can also lead to sleep disturbance and fragmented sleep. ${ }^{11}$ Experimental studies have found associations between transportation noise at night time and endothelial dysfunction, increased oxidative stress, alterations in the immune system, and increased systemic inflammation, ${ }^{12-15}$ which in turn are regarded as 
early events in the onset of dementia and Alzheimer's disease. $^{116-21}$

The scarce existing research on noise and dementia generally does not indicate associations between transportation noise and dementia risk. ${ }^{22-24}$ However, as concluded by a systematic review in 2020, the current evidence in this research area is of low quality, because of few epidemiological studies with high diversity in study design and outcome definition. ${ }^{25}$ A few studies have, however, suggested that transportation noise might lead to cognitive impairment in adults. ${ }^{26-28}$ Therefore, further high quality population based studies are required to test the hypothesis of a potential association between exposure to transportation noise and risk of dementia. ${ }^{25}$

We investigated the association between long term residential exposure to transportation noise and risk of incident dementia in a nationwide cohort including adults aged $\geq 60$ years living in Denmark. Register based individual level information was obtained on address history, socioeconomic indicators, and incident all cause dementia and dementia subtypes.

\section{Methods}

Study population

This study is based on the population of Denmark, where all residents are followed across national health and administrative registers using a unique personal identification number. ${ }^{29}$ From the Danish Civil Registration System we identified adults aged $\geq 60$ years living in Denmark between 1 January 2004 and 31 December $2017 .{ }^{30}$ Using this register, we also obtained the histories for residential addresses from 1994 until 2017, to guarantee at least 10 years of address history for participants. ${ }^{30}$ The date of baseline was either 1 January 2004 or the date at which the participants turned 60 years, whichever came last. We excluded residents with an incomplete address history in the 10 years before baseline, to ensure long term exposure for participants, and those with a dementia diagnosis before baseline. Start of follow-up was chosen as 2004, because 2003 marks a noticeable improvement in the diagnostic rate of dementia compared with previous years. $^{31}$

\section{Identification of outcomes}

We identified people with dementia by linking their personal identification numbers to nationwide health registries. All cause dementia was defined as primary or secondary diagnoses of dementia for inpatient and outpatient contacts recorded in the Danish National Patient Register or the Danish Psychiatric Central Register (method 1), ${ }^{32}$ or at least one prescription of an antidementia drug (donepezil, rivastigmine, galantamine, or memantine) registered in the Danish National Prescription Registry (method 2), or both. ${ }^{34}$ For subtype analyses, we considered specific diagnoses of Alzheimer's disease, vascular dementia, and Parkinson's disease related dementia. Supplementary table 1 lists the diagnostic codes used for all cause dementia and dementia subtypes. We defined date of diagnosis as the date of the first recorded dementia diagnosis or the date of the first prescription of an antidementia drug, whichever came first.

\section{Noise exposure assessment}

We estimated exposures to road traffic and railway noise at the most and least exposed façades of buildings for all residential addresses in Denmark. Noise levels were calculated as the equivalent continuous A-weighted sound pressure levels $\left(\mathrm{L}_{\text {Aeq }}\right)$ during the day (7 am to 7 $\mathrm{pm})$, evening (7 pm to $10 \mathrm{pm}$ ), and night (10 pm to 7 am) and are presented as $\mathrm{L}_{\text {den }}$ (ie, the average sound level over 24 hours, considering a $5 \mathrm{~dB}$ penalty for the evening and a $10 \mathrm{~dB}$ penalty for the night, to account for higher susceptibility to noise and stress in these periods)..$^{35}$

The calculations of road traffic and railway noise were based on the Nordic prediction method, ${ }^{36}$ and Nord2000 models, ${ }^{37}$ respectively, using the software SoundPLAN (version 8.0, SoundPLAN Nord ApS, Odense, Denmark). Both models are based on the same acoustical principals and have been validated previously-one study that compared measured and predicted values for 174 sites in four Nordic countries (Denmark, Sweden, Norway, and Finland), found an average difference of only $0.3 \mathrm{~dB}(\mathrm{SD} 3.0 \mathrm{~dB}){ }^{38}$

Major input variables for road traffic noise estimations were geographical address coordinates and height (floor) of each address, and traffic information extracted from a national database on, for example, annual average daily traffic (for 1995, 2000, 2005, 2010, and 2015), vehicle distribution (light, heavy), travel speed, and road type (motorway, rural highway, road $>6 \mathrm{~m}$, road 3-6 $\mathrm{m}$, and other road). ${ }^{39}$ Major input variables for the railway noise models included geocoded address data, information on annual average daily train lengths, travel speed, and train types obtained from the Danish state railway network. Both road traffic and railway models considered the screening effects from terrains (terrain information retrieved from the GeoDanmark in $2.5 \mathrm{~m}$ contour lines), buildings (three dimensional building footprints), noise barriers, and noise berms; ground absorption; and first and second order noise reflections from building façades. We estimated noise exposures at the centre of all façades for each specific address. Buildings with several address points, such as apartments and townhouses, were split into different polygons, resulting in one building polygon for each address point. The lowest and highest noise levels for each address were expressed as exposure at the most $\left(\mathrm{L}_{\mathrm{den}} \max \right)$ and least $\left(\mathrm{L}_{\mathrm{den}} \min \right)$ exposed façades, respectively.

We modelled road traffic noise for all Danish addresses for 1995, 2000, 2005, 2010, and 2015, and railway noise for all addresses within $1000 \mathrm{~m}$ of a rail track for 1997 and 2012. Based on the estimations for these five time points for road traffic noise and two time points for railway noise, we applied linear interpolation to calculate yearly noise estimates for each of the years from 1994 to 2017 for all Danish addresses. 
We subsequently linked the address specific yearly noise exposures at all Danish residential addresses to the person specific address histories obtained for the whole study population.

For each cohort member we then calculated time weighted 10 year running means for $L_{\text {den }} \max$ and $\mathrm{L}_{\text {den }}$ min, taking exposure at all addresses in the periods into account. The time weighted means were calculated as arithmetic means on the sound pressure scale followed by logarithmic transformation to the decibel scale. All noise values below $35 \mathrm{~dB}$ were then set to $35 \mathrm{~dB}$ because we do not expect people to perceive transportation noise levels below this threshold because of background noise from other sources.

\section{Covariates}

We collected a variety of covariates from registers available at Statistics Denmark: yearly individual level variables (from 2004 to 2017) on civil status (married or cohabiting, widowed, divorced, and single), country of origin (Denmark, other western country, nonwestern country), individual income (fifths, based on the yearly distribution among Danish adults aged 2570 years), occupational status (blue collar, low level white collar, high level white collar, unemployed, retired), and highest attained education (mandatory, secondary or vocational, medium or long education). Furthermore, for each of the 2160 parishes available in Denmark, we gained information on population density (inhabitants $/ \mathrm{km}^{2}$ ) and neighbourhood level socioeconomic status (ie, the proportion of residents with low income (lowest fourth), unemployed, in manual labour, with only basic education, with a criminal record, and living in a single parent household). We also extracted information on the building type (detached house, semidetached house, multistorey building, and other) for each address from the Building and Housing Register.

We calculated the proportion of high quality green space (ie, recreational areas, forests, and wet and dry nature areas) within $150 \mathrm{~m}$ radius and $1000 \mathrm{~m}$ radius of each address, using land use and land cover maps of Denmark. ${ }^{41}$ Furthermore, for all Danish addresses, we estimated concentrations of fine particulate matter $\left(\mathrm{PM}_{2.5}\right)$ and nitrogen dioxide for 2000, 2010, and 2015 using the Danish DEHM-UBM-AirGIS modelling system. Calculations in DEHM-UBM-AirGIS combine three models operating at different spatial scales (DEHM: regional background, UBM: urban background, and AirGIS: traffic in nearest streets), taking into account factors such as road and traffic information, street configurations, emissions from all Danish sources in high resolution, and background concentrations from outside Denmark. ${ }^{42}$ We subsequently calculated yearly and spatially averaged urban background $\mathrm{PM}_{2.5}$ and nitrogen dioxide levels in Denmark for 1994-2017 using the DEHM-UBM model. These yearly background means were used to calculate yearly scaling factors, which we used to extrapolate the address specific estimates from 2000, 2010, and 2015 into yearly address specific means for 1994-2017.

\section{Statistical analysis}

Cox proportional hazard models were used with age as the underlying time to calculate hazard ratios for the association between transportation noise at the most exposed façades $\left(\mathrm{L}_{\mathrm{den}} \max \right)$ and least exposed façades ( $\mathrm{L}_{\mathrm{den}}$ min) and all cause dementia and dementia subtypes. We right censored at the age of a dementia diagnosis, death, missing address, emigration, or end of follow-up (31 December 2017), whichever came first. The time weighted running means for road traffic and railway noise were included as time varying variables into the Cox model; thus for each participant with a dementia diagnosis comparing with the 10 year mean exposure for all participants without a dementia diagnosis at the same age as the participant with dementia.

To test the assumption of proportional hazards for all covariates, we evaluated the independence between scaled Schoenfeld residuals with time. We included sex, civil status, occupational status, income, and calendar year as strata, as these deviated from the assumption of proportional hazards.

We analysed the associations between $5 \mathrm{~dB}$ categories of 10 year mean road traffic and railway $\mathrm{L}_{\text {den }}$ max and $\mathrm{L}_{\text {den }}$ min and all cause dementia using three models: model 1, adjusted for age (by design), sex, and calendar year; model 2, with further adjustment for region of origin, civil status, personal income, occupational status, proportion of high quality green space, and area level socioeconomic variables, as well as mutual adjustment for road traffic and railway noise; and model 3, with additional adjustment for time weighted 10 years running means of $\mathrm{PM}_{2.5}$. We used $<45 \mathrm{~dB}$ as the reference category for all analyses with road traffic $\mathrm{L}_{\mathrm{den}} \max$, and $<40 \mathrm{~dB}$ for all analyses with road traffic $\mathrm{L}_{\mathrm{den}}$ min and railway $\mathrm{L}_{\text {den }}$ max and $\mathrm{L}_{\text {den }}$ min. Associations were also analysed between categories of 10 year mean exposure to road traffic and railway noise and dementia subtypes (Alzheimer's disease, vascular dementia, and Parkinson's disease related dementia) adjusting for the covariates in model 2.

We chose the covariates for the statistical model based on availability in national registers and a literature review of plausible mechanisms behind a potential association between transportation noise and dementia, as shown by the directed acyclic graph (supplementary figure 1). A priori we selected model 2 as our main adjustment model. All covariates, apart from sex and region of origin, were included in the statistical models as time varying variables, therefore allowing for change throughout the follow-up period.

Natural cubic splines with four degrees of freedom (based on adjustment of model 2) were applied to investigate the shape of the exposure-response association between road traffic and railway noise $\left(\mathrm{L}_{\mathrm{den}} \max\right.$ and $\left.\mathrm{L}_{\text {den }} \min \right)$ and risk of all cause dementia, and between road traffic noise $\left(\mathrm{L}_{\mathrm{den}} \max\right.$ and $\left.\mathrm{L}_{\mathrm{den}} \min \right)$ and dementia subtypes (Alzheimer's disease, vascular dementia, and Parkinson's disease related dementia).

The joint effect of exposure to road traffic and railway noise in relation to all cause dementia was 
investigated by combining three categories of road traffic noise $\left(\mathrm{L}_{\mathrm{den}}\right.$ max of $<55 \mathrm{~dB}, 55-65 \mathrm{~dB}$, and $\geq 65$ $\mathrm{dB}$, and $\mathrm{L}_{\mathrm{den}}$ min of $<45 \mathrm{~dB}, 45-55 \mathrm{~dB}$, and $\geq 55 \mathrm{~dB}$ ) and three categories of railway noise $\left(\mathrm{L}_{\mathrm{den}} \max\right.$ of $<40 \mathrm{~dB}$, 40-50 dB, and $\geq 50 \mathrm{~dB}$, and $\mathrm{L}_{\mathrm{den}}$ min of $<35 \mathrm{~dB}, 35-45$ $\mathrm{dB}$, and $\geq 45 \mathrm{~dB}$ ) using low road traffic and railway noise as reference. Similarly, we investigated the joint effect of exposure to road traffic noise at the most and least exposed building façades in relation to all cause dementia by combining three categories of $\mathrm{L}_{\mathrm{den}} \max$ $(<55 \mathrm{~dB}, 55-60 \mathrm{~dB}$, and $\geq 60 \mathrm{~dB})$ and three categories of $\mathrm{L}_{\mathrm{den}} \min (<40 \mathrm{~dB}, 40-50 \mathrm{~dB}$, and $\geq 50 \mathrm{~dB}$ ) using low $\mathrm{L}_{\mathrm{den}} \max$ and $\mathrm{L}_{\mathrm{den}} \min$ as reference.

For all cause dementia, we conducted sensitivity analyses based on model 2 by including both $L_{\text {den }} \max$ and $\mathrm{L}_{\mathrm{den}}$ min; adjusting for both time weighted exposures to $\mathrm{PM}_{2.5}$ and nitrogen dioxide; further adjusting for time varying highest attained education for the subpopulation for which this information was available; and including only cases with a primary dementia diagnosis. We also investigated associations with categories of 10 year time weighted night time noise $\left(\mathrm{L}_{\text {night }}\right)$.

We evaluated possible effect modification of the association between 10 year mean road traffic noise at the least exposed façade and all cause dementia by generating natural cubic splines with four degrees of freedom (based on adjustment of model 2) for each of the following groups: men and women; low, medium, and high population density; detached house, semidetached house, and multistorey building; low, medium, and high income; before and after 2010; and with and without comorbidity from myocardial infarction, stroke, or diabetes.

All statistical analyses were performed in SAS 9.4 (SAS Institute, Cary, NC), apart from the analysis of proportional hazards and natural splines, for which we used R, version 3.6.3 (survival package).

\section{Patient and public involvement}

As this is a register based study, no patients or members of the public were involved in the design, conduct, reporting, or dissemination plans of our research.

\section{Results}

The study base included 2.1 million Danish residents aged $\geq 60$ years. People were excluded if they had a diagnosis of dementia before baseline $(n=87109)$, had an incomplete history on exposure before enrolment $(n=69692)$, or were missing covariates $(n=2411)$, resulting in a study population of 1938994 participants and 103500 participants with incident dementia. The mean follow-up was 8.5 years (ie, a total of 16425922 person years), with an event rate of 6.3 cases/1000 person years. Of the participants with dementia, 18628 were exclusively identified by method 2 (supplementary figure 2), and 1899 (<2\% of participants with dementia) were identified based on less than three prescriptions for antidementia drugs. In total, 31219 participants received a diagnosis of Alzheimer's disease, 8664 a diagnosis of vascular dementia, and 2192 a diagnosis of Parkinson's disease related dementia. The incidence rate of dementia in the study population was stable during the study period, with, for example, 7282 participants identified with dementia in 2004 and 7058 participants identified with dementia in 2017.

Table 1 and supplementary table 2 show the baseline characteristics of the study population for road traffic $\mathrm{L}_{\mathrm{den}} \max$ below and above $55 \mathrm{~dB}$ and for participants with or without dementia. The median 10 year exposure was $55.3 \mathrm{~dB}\left(\mathrm{~L}_{\mathrm{den}} \max \right)$ and $44.3 \mathrm{~dB}$ $\left(\mathrm{L}_{\mathrm{den}} \min \right)$ for road traffic noise, and $51.6 \mathrm{~dB}\left(\mathrm{~L}_{\mathrm{den}} \max \right)$ and $44.7 \mathrm{~dB}\left(\mathrm{~L}_{\mathrm{den}} \mathrm{min}\right)$ for railway noise (among those who were exposed). Supplementary figures 3 and 4 show the distribution of exposure to road traffic and railway noise at baseline, and supplementary figure 5 shows the distribution of the difference between noise measured at the most and least exposed façades.

For road $\mathrm{L}_{\mathrm{den}}$ max, Spearman correlation coefficients of 0.49 were found for road $\mathrm{L}_{\mathrm{den}} \min , 0.12$ for railway $\mathrm{L}_{\text {den }} \max , 0.10$ for railway $\mathrm{L}_{\text {den }} \min , 0.25$ for $\mathrm{PM}_{2.5}$, and 0.45 for nitrogen dioxide. Only weak correlations were observed between these variables and proportion of high quality green space (supplementary table 3). Results also showed high correlations between $\mathrm{L}_{\mathrm{den}}$ and $\mathrm{L}_{\text {night }}$, especially for road traffic noise (about 1.00 for most and least exposed façades), but also for railway noise (0.94 for $\mathrm{L}_{\mathrm{den}} \max$ and 0.83 for $\mathrm{L}_{\mathrm{den}} \min$ ).

Road traffic and railway noise at the most and least exposed façade were associated with increased risk of all cause dementia across all exposure categories (table 2) with, for example, hazard ratios of 1.16 (95\% confidence interval 1.13 to 1.19) for road $\mathrm{L}_{\mathrm{den}} \max \geq 65$ $\mathrm{dB}$ (compared with $<45 \mathrm{~dB}$ ), 1.18 (1.15 to 1.21) for road $\mathrm{L}_{\mathrm{den}} \min \geq 55 \mathrm{~dB}$ (compared with $<40 \mathrm{~dB}$ ), 1.16 (1.12 to 1.20) for railway $L_{\text {den }} \max \geq 60 \mathrm{~dB}$ (compared with $<40$ $d B$ ), and 1.16 (1.12 to 1.19) for railway $L_{d e n} \min \geq 50$ $\mathrm{dB}$ (compared with $<40 \mathrm{~dB}$ ). The associations for road traffic noise followed exposure-response associations until about $55 \mathrm{~dB}$ for $\mathrm{L}_{\mathrm{den}} \max$ and $50 \mathrm{~dB}$ for $\mathrm{L}_{\mathrm{den}}$ min, after which no further increase or even a slight decrease in risk was observed (fig 1). Hazard ratios were slightly higher for $\mathrm{L}_{\mathrm{den}}$ min compared with $\mathrm{L}_{\mathrm{den}}$ max. Similarly, a positive exposure-response association was found for railway noise $\mathrm{L}_{\mathrm{den}}$ max, with a slight decline in risk at higher exposures (fig 1). For railway $\mathrm{L}_{\mathrm{den}} \mathrm{min}$, however, an upward trend was observed until about $45 \mathrm{~dB}$, with a levelling off in risk at medium exposure levels ( $45 \mathrm{~dB}$ to $55 \mathrm{~dB}$ ), followed by an increase in risk from $55 \mathrm{~dB}$. Associations between exposure to noise at night time $\left(\mathrm{L}_{\text {night }}\right)$ and all cause dementia were generally similar to the associations observed for $\mathrm{L}_{\mathrm{den}}$ (supplementary table 4).

Based on the hazard ratios and the percentage of exposed people in each noise category, the population attributable risk for road traffic and railway noise was calculated. Of the 8475 patients with incident dementia registered in Denmark in $2017,{ }^{43}$ the diagnosis in an estimated 963 patients (95\% confidence interval 762 to 1174) was attributed to road traffic noise and in 253 patients (195 to 310) to railway noise. 


\begin{tabular}{|c|c|c|}
\hline \multicolumn{3}{|c|}{$\begin{array}{l}\text { Table } 1 \text { | Characteristics of study population at baseline }(\mathrm{n}=1938994) \text { according to } \\
\text { exposure to road traffic noise at most exposed façade of building. Values are numbers } \\
\text { (percentages) unless stated otherwise }\end{array}$} \\
\hline \multirow{2}{*}{ Characteristics } & \multicolumn{2}{|l|}{ Exposure level } \\
\hline & $\langle 55 \mathrm{~dB}(\mathrm{n}=946609)$ & $\geq 55 \mathrm{~dB}(\mathrm{n}=992385)$ \\
\hline \multicolumn{3}{|l|}{ Individual level } \\
\hline Men & $449413(47.5)$ & $458578(46.2)$ \\
\hline Mean (SD) age (years) & $65.8(8.2)$ & $66.5(8.8)$ \\
\hline \multicolumn{3}{|l|}{ Country of origin: } \\
\hline Denmark & $937329(99.0)$ & $976742(98.4)$ \\
\hline Other western country & $5310(0.6)$ & $7328(0.7)$ \\
\hline Non-western country & $3970(0.4)$ & $8315(0.9)$ \\
\hline \multicolumn{3}{|l|}{ Civil status: } \\
\hline Married or cohabiting & $695872(73.5)$ & $644905(65.0)$ \\
\hline Widowed & $124856(13.2)$ & $152267(15.3)$ \\
\hline Divorced & $74634(7.9)$ & $118670(12.0)$ \\
\hline Single & $51247(5.4)$ & $76543(7.7)$ \\
\hline \multicolumn{3}{|l|}{ Individual income fifth*: } \\
\hline 1st (lowest) & $260210(27.5)$ & $305196(30.7)$ \\
\hline 2nd & $219369(23.1)$ & $247982(25.0)$ \\
\hline $3 \mathrm{rd}$ & $149286(15.8)$ & $151689(15.3)$ \\
\hline 4th & $147421(15.6)$ & $134824(13.6)$ \\
\hline 5th (highest) & $170323(18.0)$ & $152694(15.4)$ \\
\hline \multicolumn{3}{|l|}{ Occupational status: } \\
\hline Blue collar & $85731(9.1)$ & $75598(7.6)$ \\
\hline White collar & $346480(36.6)$ & $336163(33.9)$ \\
\hline Unemployed & $36472(3.8)$ & $43324(4.4)$ \\
\hline Retired & $477926(50.5)$ & $537300(54.1)$ \\
\hline \multicolumn{3}{|l|}{ Highest attained education: } \\
\hline Mandatory education & $343531(36.3)$ & $375759(37.9)$ \\
\hline Secondary or vocational education & $373875(39.5)$ & $373290(37.6)$ \\
\hline Medium or long education & $166392(17.6)$ & $158986(16.0)$ \\
\hline Unknown & $62811(6.6)$ & $84350(8.5)$ \\
\hline \multicolumn{3}{|l|}{ Address levelt } \\
\hline \multicolumn{3}{|l|}{ Proportion of high quality green space: } \\
\hline$\geq 15 \%$ in $150 \mathrm{~m}$ radius & $207299(21.9)$ & $201448(20.3)$ \\
\hline$\geq 20 \%$ in $1000 \mathrm{~m}$ radius & $259355(27.4)$ & $239881(24.2)$ \\
\hline \multicolumn{3}{|l|}{ Urbanity (inhabitants $/ \mathrm{km}^{2}$ ): } \\
\hline Low $(\leq 100)$ & $248130(26.2)$ & $252827(25.5)$ \\
\hline Medium (101-4999) & $673684(71.2)$ & $640993(64.6)$ \\
\hline High (25000) & $24795(2.6)$ & $98565(9.9)$ \\
\hline \multicolumn{3}{|l|}{ Building type: } \\
\hline Detached house & $633563(66.9)$ & $500529(50.4$ \\
\hline Semidetached house & $162122(17.1)$ & $141659(14.3)$ \\
\hline Multistorey building & $149096(15.8)$ & $347269(35.0)$ \\
\hline Other & $1828(0.2)$ & $2928(0.3)$ \\
\hline \multicolumn{3}{|c|}{ Mean (SD) 10 year mean air pollution exposure $\left(\mu \mathrm{g} / \mathrm{m}^{3}\right)$ : } \\
\hline $\mathrm{PM}_{2.5}$ & $15.2(1.7)$ & $15.9(2.1)$ \\
\hline Nitrogen dioxide & $16.5(5.2)$ & $21.3(8.7)$ \\
\hline \multicolumn{3}{|c|}{ Mean (SD) area level factors (\% of population): } \\
\hline Low income (1st fourth) & $4.4(2.0)$ & $5.0(2.4)$ \\
\hline Unemployed & $1.7(0.7)$ & $1.8(0.7)$ \\
\hline Manual labour & $13.1(3.1)$ & $12.2(3.3)$ \\
\hline Only basic education & $10.6(3.4)$ & $10.2(3.5)$ \\
\hline Criminal record & $0.4(0.3)$ & $0.5(0.3)$ \\
\hline Single parent families & $5.6(1.7)$ & $5.7(1.8)$ \\
\hline
\end{tabular}

$\mathrm{PM}_{2.5}=$ fine particulate matter.

*Standardised by calendar year and sex.

tBased on residential address of participants at baseline.

Higher risk estimates for Alzheimer's disease were found for both road traffic and railway noise, with the highest risk estimates for road traffic $\mathrm{L}_{\mathrm{den}} \min$ (hazard ratio $1.30,95 \%$ confidence interval 1.25 to 1.36 ) for the exposure category $50-55 \mathrm{~dB}$ and 1.27 (1.22 to 1.34) for $\geq 55 \mathrm{~dB}$, compared with $<40 \mathrm{~dB}$; table 2). No clear associations were found between exposure to railway noise and vascular dementia. Furthermore, no associations were observed between road traffic $\mathrm{L}_{\mathrm{den}} \max$ and risk of Parkinson's disease related dementia, whereas some indication of an association was found for road traffic $\mathrm{L}_{\mathrm{den}} \mathrm{min}$. However, only a few participants had Parkinson's disease related dementia and therefore confidences were wide for this outcome. Exposure-response associations for road $\mathrm{L}_{\mathrm{den}} \min$ and all dementia subtypes followed an upward trend until about $55 \mathrm{~dB}$ for Alzheimer's disease, $50 \mathrm{~dB}$ for vascular dementia, and $53 \mathrm{~dB}$ for Parkinson's disease related dementia. After these thresholds, we observed a decrease in hazard ratios, however with wide confidence intervals (fig 2). Splines for $\mathrm{L}_{\mathrm{den}}$ max showed increases in risk for all subtypes until approximately $55 \mathrm{~dB}$ for Alzheimer's disease, and $60 \mathrm{~dB}$ for vascular dementia and Parkinson's disease related dementia, after which a levelling off in risk was observed.

When investigating associations between categories combining road traffic $\mathrm{L}_{\mathrm{den}}$ max and $\mathrm{L}_{\mathrm{den}} \min$ in relation to all cause dementia, exposure-response associations were observed for $\mathrm{L}_{\text {den }}$ min in all exposure categories of $\mathrm{L}_{\mathrm{den}}$ max, whereas the opposite was not observed (supplementary table 5). In an analysis combining categories of road traffic and railway noise, coexposure to medium-high levels of both road traffic and railway noise was associated with high hazard ratios-for example, for road traffic $\mathrm{L}_{\mathrm{den}} \max \geq 65 \mathrm{~dB}$ and railway $\mathrm{L}_{\mathrm{den}} \max \geq 50 \mathrm{~dB}$ the hazard ratio was 1.22 (95\% confidence interval 1.18 to 1.27 ; supplementary table 6).

Adjustment for air pollution $\left(\mathrm{PM}_{2.5}\right.$ alone and together with nitrogen dioxide) and education resulted in some reductions in continuous hazard ratios for all cause dementia, although associations with both road traffic and railway noise remained (supplementary tables 7 and 8). Mutual adjustment for $\mathrm{L}_{\text {den }} \max$ and $\mathrm{L}_{\mathrm{den}}$ min was not associated with substantial changes in risk estimates (supplementary table 10). When restricting analyses to participants with a primary diagnosis of dementia $(n=42413)$, the hazard ratios for road traffic and railway $\mathrm{L}_{\mathrm{den}}$ min categories were observed to increase-for example, for the highest exposure category $(\geq 55 \mathrm{~dB}$ for road traffic and $\geq 50$ $\mathrm{dB}$ for railway) compared with the reference group increased from 1.18 (1.15 to 1.21 ) to 1.30 (1.25 to 1.35) for road traffic noise and from 1.16 (1.12 to 1.19) to 1.21 (1.16 to 1.27 ) for railway noise (supplementary table 8). No substantial increase in hazard ratios was, however, observed for railway and road traffic $\mathrm{L}_{\mathrm{den}} \max$.

Figure 3 presents splines for the associations between 10 year mean road traffic noise $\left(\mathrm{L}_{\mathrm{den}} \min \right)$ and all cause dementia in relation to potential effect modifiers. Stronger associations were found between road traffic noise and dementia among people living in low compared with medium and high populated areas, and among people living in detached houses versus semidetached houses and multistorey buildings. Even though the curves overlapped, hazard ratios were found to be slightly lower for people with higher income compared with those with lower or medium income. No substantial differences were observed 


\begin{tabular}{|c|c|c|c|c|c|c|c|c|}
\hline \multirow[b]{2}{*}{$\begin{array}{l}\text { Noise exposure } \\
\text { (10 year) by category }\end{array}$} & \multicolumn{2}{|c|}{ All cause dementia } & \multicolumn{2}{|c|}{ Alzheimer's disease } & \multicolumn{2}{|c|}{ Vascular dementia } & \multicolumn{2}{|c|}{ Parkinson's disease dementia } \\
\hline & No of cases & $\begin{array}{l}\text { Hazard ratio } \\
(95 \% \mathrm{Cl})^{\star}\end{array}$ & No of cases & $\begin{array}{l}\text { Hazard ratio } \\
(95 \% \mathrm{Cl})^{\star}\end{array}$ & No of cases & $\begin{array}{l}\text { Hazard ratio } \\
(95 \% \mathrm{Cl})^{\star}\end{array}$ & No of cases & $\begin{array}{l}\text { Hazard ratio } \\
(95 \% \mathrm{Cl})^{*}\end{array}$ \\
\hline \multicolumn{9}{|l|}{ Road } \\
\hline \multicolumn{9}{|l|}{$\mathrm{L}_{\mathrm{den}} \max (\mathrm{dB}):$} \\
\hline «45 (reference) & 9718 & 1 & 2799 & 1 & 827 & 1 & 242 & 1 \\
\hline $45-50$ & 13500 & 1.09 (1.06 to 1.12$)$ & 4081 & 1.10 (1.04 to 1.15$)$ & 1090 & 1.03 (0.94 to 1.13$)$ & 287 & $0.94(0.79$ to 1.12$)$ \\
\hline $50-55$ & 21738 & $1.16(1.13$ to 1.19$)$ & 6798 & $1.19(1.14$ to 1.25$)$ & 1754 & $1.10(1.01$ to 1.20$)$ & 463 & $1.03(0.88$ to 1.21$)$ \\
\hline $55-60$ & 24533 & 1.17 (1.14 to 1.20$)$ & 7427 & $1.17(1.12$ to 1.22$)$ & 2084 & 1.17 (1.08 to 1.27$)$ & 519 & $1.07(0.92$ to 1.25$)$ \\
\hline $60-65$ & 21196 & 1.16 (1.13 to 1.19$)$ & 6242 & 1.14 (1.09 to 1.19$)$ & 1802 & 1.18 (1.08 to 1.28$)$ & 443 & $1.10(0.94$ to 1.29$)$ \\
\hline$\geq 65$ & 12815 & 1.16 (1.13 to 1.19$)$ & 3872 & 1.16 (1.11 to 1.22$)$ & 1107 & 1.19 (1.09 to 1.31$)$ & 238 & $1.04(0.87$ to 1.25$)$ \\
\hline \multicolumn{9}{|l|}{$\mathrm{L}_{\mathrm{den}} \min (\mathrm{dB})$} \\
\hline ८40 (reference) & 18136 & 1 & 4902 & 1 & 1475 & 1 & 394 & 1 \\
\hline $40-45$ & 30306 & $1.12(1.10$ to 1.14$)$ & 8608 & 1.10 (1.06 to 1.14$)$ & 2621 & 1.19 (1.12 to 1.27$)$ & 619 & 1.10 (0.96 to 1.25$)$ \\
\hline $45-50$ & 27261 & 1.18 (1.16 to 1.20$)$ & 8403 & $1.22(1.17$ to 1.26$)$ & 2239 & $1.20(1.12$ to 1.28$)$ & 580 & 1.18 (1.03 to 1.35$)$ \\
\hline $50-55$ & 18005 & 1.21 (1.19 to 1.24$)$ & 5985 & $1.30(1.25$ to 1.36$)$ & 1567 & 1.31 (1.21 to 1.41$)$ & 402 & $1.26(1.08$ to 1.46$)$ \\
\hline$\geq 55$ & 9792 & $1.18(1.15$ to 1.21$)$ & 3321 & $1.27(1.22$ to 1.34$)$ & 762 & $1.14(1.04$ to 1.25$)$ & 197 & $1.16(0.96$ to 1.39$)$ \\
\hline \multicolumn{9}{|l|}{ Railway } \\
\hline \multicolumn{9}{|l|}{$\mathrm{L}_{\mathrm{den}} \max (\mathrm{dB}):$} \\
\hline ८40 (reference) & 75690 & 1 & 22819 & 1 & 6409 & 1 & 1603 & 1 \\
\hline $40-45$ & 4441 & 1.09 (1.06 to 1.12$)$ & 1287 & $1.02(0.96$ to 1.08$)$ & 387 & $1.14(1.03$ to 1.26$)$ & 117 & $1.43(1.18$ to 1.72$)$ \\
\hline $45-50$ & 5844 & 1.11 (1.08 to 1.14$)$ & 1737 & $1.06(1.01$ to 1.12$)$ & 488 & $1.10(1.00$ to 1.20$)$ & 122 & $1.17(0.97$ to 1.41$)$ \\
\hline $50-55$ & 8040 & 1.19 (1.16 to 1.22$)$ & 2491 & $1.20(1.15$ to 1.26$)$ & 656 & $1.13(1.04$ to 1.23$)$ & 149 & $1.16(0.98$ to 1.38$)$ \\
\hline $55-60$ & 5577 & 1.19 (1.16 to 1.23$)$ & 1695 & $1.20(1.15$ to 1.27$)$ & 403 & $0.99(0.90$ to 1.10$)$ & 134 & 1.49 (1.25 to 1.78$)$ \\
\hline$\geq 60$ & 3908 & $1.16(1.12$ to 1.20$)$ & 1190 & $1.16(1.10$ to 1.23$)$ & 321 & 1.09 (0.97 to 1.22$)$ & 67 & 1.07 (0.84 to 1.37$)$ \\
\hline \multicolumn{9}{|l|}{$\mathrm{L}_{\mathrm{den}} \min (\mathrm{dB}):$} \\
\hline ८40 (reference) & 86286 & 1 & 25808 & 1 & 7309 & 1 & 1845 & 1 \\
\hline $40-45$ & 6049 & $1.12(1.09$ to 1.15$)$ & 1866 & $1.12(1.06$ to 1.17$)$ & 505 & $1.10(1.00$ to 1.20$)$ & 149 & 1.39 (1.17 to 1.64$)$ \\
\hline $45-50$ & 6302 & $1.17(1.14$ to 1.20$)$ & 1973 & $1.20(1.15$ to 1.26$)$ & 467 & $1.01(0.92$ to 1.11$)$ & 102 & $0.98(0.80$ to 1.20$)$ \\
\hline$\geq 50$ & 4863 & 1.16 (1.12 to 1.19$)$ & 1572 & 1.24 (1.17 to 1.30$)$ & 383 & 1.03 (0.93 to 1.14$)$ & 96 & $1.19(0.96$ to 1.47$)$ \\
\hline
\end{tabular}
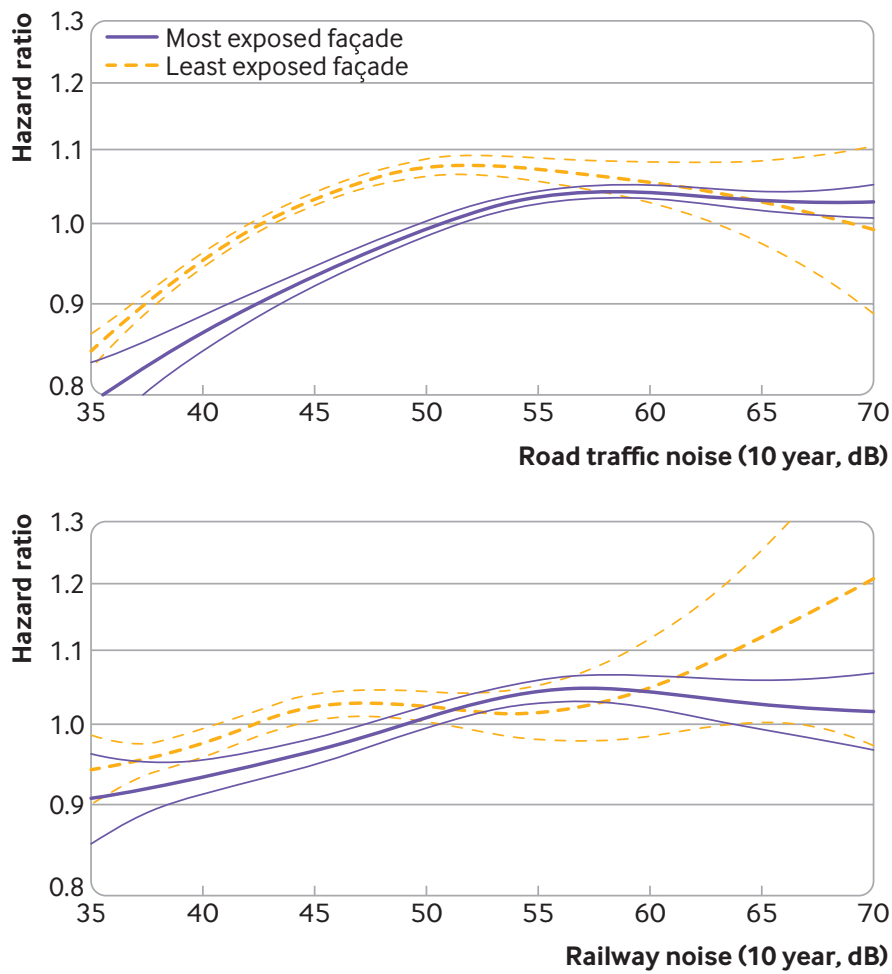

Fig 1 | Associations between 10 year mean exposure to road traffic and railway noise at the most $\left(\mathrm{L}_{\mathrm{den}} \max \right)$ and least $\left(\mathrm{L}_{\mathrm{den}} \mathrm{min}\right)$ exposed façades of buildings and risk of all cause dementia, using the fully adjusted model (model 2). Figure shows hazard ratios and corresponding $95 \%$ confidence intervals in the exposure-response curves in relation to sex, comorbidity, or calendar year. In a subanalysis, positive associations were observed between road traffic noise and all cause dementia for all five regions of Denmark (supplementary figure 6).

\section{Discussion}

In our large nationwide cohort, we found long term residential exposure to road traffic and railway noise to be associated with increased risk of all cause dementia. These associations followed positive exposure-response associations, with a levelling off or slight decreases in risk at high noise exposures for most of the exposure metrics. For Alzheimer's disease we observed associations with both road traffic and railway noise, whereas for vascular dementia we only observed clear associations for road traffic noise. We found indications of slightly higher risk estimates for 10 year mean exposure to road traffic noise at the least exposed façades of buildings ( $\mathrm{L}_{\mathrm{den}} \mathrm{min}$ ) compared with most exposed ( $\left.\mathrm{L}_{\text {den }} \max \right)$ for both Alzheimer's disease and vascular dementia. The association between road traffic noise and dementia differed in relation to population density, building type, and income.

\section{Strengths and limitations of this study}

One of the major strengths of our study is the nationwide prospective design, which included a large population, long follow-up, and high quality assessment of noise 



Fig 2 | Associations between 10 year mean exposure to road traffic noise at the most and least exposed façades of buildings and risk of dementia subtypes, using the fully adjusted model (model 2). Figure shows hazard ratios and corresponding $95 \%$ confidence intervals counts and distribution, and lack of information on other noise sources. Such misclassification is probably non-differential, which would tend to drive the risk estimates towards 1 . Also, we lacked information on noise sources that might mask the association between road traffic and railway noise and dementia, such as airports and construction sites.

Although we used three high quality nationwide registries to identify participants with dementia, we cannot rule out that outcome misclassification was a problem, mainly for definition of subtypes. One study found a low validity for register based diagnosis of dementia subtypes in Denmark, apart from Alzheimer's disease, and therefore these results should be interpreted with caution. ${ }^{44}$ Moreover, identification of participants with dementia based entirely on prescription of antidementia drugs (method 2) has not been previously validated. Diagnosing dementia is a complicated and non-standardised procedure, with potential differences in patients' socioeconomic status, and regional differences in diagnosis rates and services. ${ }^{31} 4849$ In a subanalysis, however, we found consistent associations between road traffic noise and all cause dementia for the five regions of Denmark, apart from slightly lower hazard ratios for the capital region, suggesting that regional differences in the diagnosis of dementia did not drive the observed associations in the present study.

\section{Comparison with other studies and discussion of main findings}

We found consistent associations between transportation noise from two independent noise sources (road traffic and railway) and risk of dementia. These associations became stronger when only participants with a primary diagnosis of dementia were considered. Previous research in this area is scarce and diverse, as pointed out by a recent systematic review on transportation noise and mental health. ${ }^{25}$ To the extent of our knowledge, only three studies investigated transportation noise and the incidence of dementia. The first study was based on a cohort of about 130000 adults living in London and identified participants with all cause dementia, Alzheimer's disease, and vascular dementia over a nine year period through medical records. ${ }^{23}$ The second study used a sample of about 678000 Canadian residents and identified cases of non-Alzheimer's disease dementia and Alzheimer's disease in three administrative health databases. ${ }^{24}$ That study, however, found a low number of participants with Alzheimer's disease (6.5\%), suggesting that outcome misclassification might have been a problem. The third study was based on a population of 1721 adults in Sweden, with assessment of dementia (Alzheimer's disease and vascular dementia) using a three phase procedure of testing, interview, and examination by a specialist. ${ }^{22}$ In all the studies, researchers found no indications of an association between transportation noise and risk of dementia. In contrast with our study, where we had exact information on the address location 



Fig 3 | Associations between 10 year mean exposure to road traffic noise at the least exposed façade of buildings and risk of incident all cause dementia according to sex, population density, type of building, income, calendar year, and comorbidity. Figure shows hazard ratios and corresponding $95 \%$ confidence intervals

(including floor) of all participants, the English and Canadian studies estimated road traffic noise levels at postal code level, ${ }^{23}{ }^{24}$ which increases the chance of exposure misclassification. ${ }^{50}$ The Swedish study used dichotomised exposure levels, reducing variability in the exposure assessment. ${ }^{22}$ Inaccuracy on the noise estimates in these studies might therefore have driven the risk estimates towards the null. A cross sectional German study of 2050 adults, however, did find positive associations between road traffic noise and overall mild cognitive impairment and amnestic mild cognitive impairment, assessed by five neuropsychological subtests. ${ }^{27}$ In support, a small study ( $\mathrm{n}=1612$ ) in the US found positive associations between road traffic noise and cognitive impairment or dementia, especially at noise levels $\geq 75 \mathrm{~dB}$. ${ }^{28}$ Both of these studies had the advantage of relying on residence specific assessment of noise exposure. However, similar to the study in Sweden ${ }^{22}$ and London, ${ }^{23}$ the researchers only estimated noise at the baseline address, therefore not taking residential mobility and long term exposure before diagnosis into account, as we did in our study.

The Lancet Commission report on dementia recently added air pollution as a modifiable risk factor for dementia, ${ }^{1}$ owing to the amount of extant research showing air pollution to contribute to an increased risk of dementia. ${ }^{35152}$ Air pollution and transportation noise share a common source (ie, transportation), and therefore including high quality estimations of air pollution in our models is important to disentangle the effect of the two exposures. ${ }^{53}$ Our results were robust to adjustment for air pollution, thus indicating an independent effect of noise on the biological mechanisms leading to dementia.

Most studies on transportation noise and health have assessed noise only at the most exposed façade of a building. In our study we also assessed exposure at the least exposed façade, which we hypothesise to better describe noise exposure during sleep, given that bedrooms are often located at quiet sides of the buildings. ${ }^{54}$ Several studies have suggested that night 
time noise is crucial to the adverse health effects of noise, especially from interrupted sleep, which is a critical period for mental and cognitive restoration. ${ }^{1455}$ Furthermore, an increasing body of evidence suggests associations between sleep disturbances, such as sleep quality and duration, with increased risk of all cause dementia and Alzheimer's disease. ${ }^{1}$ In our study, we found essentially the same risk estimates for whole day noise and only night time noise because of high correlations between both metrics. However, our results indicated slightly higher risk estimates for road traffic $\mathrm{L}_{\mathrm{den}}$ min compared with $\mathrm{L}_{\text {den }}$ max, especially for Alzheimer's disease and vascular dementia, but also for all cause dementia. This was supported by the analysis of combined effects of $L_{\text {den }}$ max and $L_{\text {den }}$ min, which showed exposure-response associations for $\mathrm{L}_{\mathrm{den}} \min$ in all exposure categories of $\mathrm{L}_{\mathrm{den}} \max$, whereas the opposite was not observed. This suggests that effects of noise on sleep might play an important role in increasing risk of dementia, which corroborates with previous studies on sleep quality and dementia. ${ }^{16-18}$ Also, one study found more pronounced associations between night time noise and mild cognitive impairment compared with exposure to noise during the whole day. ${ }^{27}$

When we investigated the association between noise and dementia subtypes, we found the highest and most consistent risk estimates for Alzheimer's disease. Although much is to be explored about the underlying mechanisms in Alzheimer's disease, several animal studies have shown chronic exposure to noise to exacerbate age related changes and activate the formation of key role genes that potentially act as mediators for pathological changes related to Alzheimer's disease in the hippocampus of mice. ${ }^{56-59}$ Because Parkinson' disease related dementia shares similar pathology to Alzheimer's disease (eg, accumulation of amyloid $\beta$ plaques and formation of tau hyperphosphorylation in the brain), and both diseases can often coexist, ${ }^{60}$ we expected compatible results for both Alzheimer's disease and Parkinson's disease related dementia. Nevertheless, results were not so consistent, especially for railway noise.

Previous studies found transportation noise to be associated with increased risk of stroke, ${ }^{68}$ which corroborates our results of an association between road traffic noise and vascular dementia, as vascular dementia is caused by poor blood flow to the brain. ${ }^{61}$ In agreement with our findings, a recent nationwide Danish study found road traffic noise to increase the risk of incident stroke, whereas no clear associations were observed for railway noise. ${ }^{62}$ Studies on exposure to transportation noise and stroke are not, however, consistent, ${ }^{63} 64$ thus indicating a need for further studies.

For most of the associations, we observed a levelling off or decline in hazard ratios at high levels of noise, especially for road traffic noise, potentially related to investments in better sound insulation at higher noise levels. ${ }^{5465}$ In support, we observed lower risk estimates among residents living in medium and highly populated areas and living in the capital region, which we would expect to be groups prioritising such measures. In line with our findings, previous studies on transportation noise and mortality also estimated higher risks in suburban areas compared with urban areas. ${ }^{5066}$ Furthermore, exposure misclassification is expected to be higher in densely populated areas, as inner city areas with narrow streets and high buildings tend to have higher estimation error as a result of many reflections, and our model includes only first and second order reflections. Besides, traffic congestion is more common in highly populated areas, which results in higher noise levels not accounted for by the noise model. Lastly, the presence of many competing noise sources in highly populated areas from, for example, community life, might lead to masking of the association between transportation noise and dementia. ${ }^{50}$ In line with this, we found lower hazard ratios for residents living in multistorey buildings and semidetached houses compared with detached houses. Other potential explanations for this levelling off might be a potential survivor effect, as residents who are less tolerant to noise might have moved away from highly exposed areas or died from competing diseases related to noise, such as cardiovascular diseases. At present, however, it is unclear whether people with a low tolerance to noise are more susceptible to health effects of noise, as findings on effect modification by noise annoyance are inconsistent. ${ }^{67-69}$

\section{Conclusions}

In this large nationwide cohort study, we found transportation noise from road traffic and railway to be associated with increased risk of all cause dementia and dementia subtypes, especially Alzheimer's disease. If these findings are confirmed in future studies, they might have a large effect on the estimation of the burden of disease and healthcare costs attributed to transportation noise. Expanding our knowledge on the harmful effects of noise on health is essential for setting priorities and implementing effective policies and public health strategies focused on the prevention and control of diseases, including dementia.

\section{AUTHOR AFFILIATIONS}

${ }^{1}$ The Mærsk McKinney Møller Institute, University of Southern Denmark, Odense, Denmark

${ }^{2}$ Diet, Genes and Environment, Danish Cancer Society Research Center, Copenhagen, Denmark

${ }^{3}$ Department of Public Health, The Research Unit for General Practice and Section of General Practice, University of Copenhagen, Copenhagen, Denmark

${ }^{4}$ Research Unit of General Practice, Department of Public Health, University of Southern Denmark, Odense, Denmark

${ }^{5}$ Department of Neurology, Slagelse Hospital, Slagelse, Denmark ${ }^{6}$ Department of Regional Health Research, University of Southern Denmark, Odense, Denmark

${ }^{7}$ Department of Environmental Science, Aarhus University, Roskilde, Denmark

${ }^{8}$ Global Centre for Clean Air Research (GCARE), University of Surrey, Guildford, United Kingdom

${ }^{9}$ Danish Big Data Centre for Environment and Health (BERTHA), Aarhus University, Roskilde, Denmark

${ }^{10}$ Research Unit for ORL - Head and Neck Surgery and Audiology, Odense University Hospital, Odense, Denmark 
${ }^{11}$ Department of Clinical Research, University of Southern Denmark, Odense, Denmark

${ }^{12}$ OPEN, Odense Patient data Explorative Network, Odense University Hospital, Odense, Denmark

${ }^{13}$ BRIDGE, Brain Research - Inter-Disciplinary Guided Excellence, Department of Clinical Research, Faculty of Health Sciences, University of Southern Denmark, Odense, Denmark

${ }^{14}$ Department of Natural Science and Environment, Roskilde University, Roskilde, Denmark

Contributors: MS, MLC, JHS, FBW, LW, ERP, OR-N, JDT, and

AHP conceived and designed the study and contributed to the methodology definition. JDT, AHP, MS, and OR-N contributed to exposure assessment. MK and JK geocoded addresses and estimated air pollution. VHV geocoded addresses and estimated proportion of green space. MS, OR-N, and AHP acquired health and confounder data. MLC classified register data and conducted the statistical analysis. MLC and MS wrote the first draft of the manuscript. Al authors commented and contributed to the interpretation of results and the final manuscript. The corresponding author attests that all listed authors meet the authorship criteria.

Funding: This work was funded by the William Demant Foundation (grant No: 18-0964) and the Independent Research Fund Denmark (grant No: 7016-00036B). The funders had no role in the design and conduct of the study; collection, analysis, and interpretation of data, writing of the manuscript, and decision to submit the manuscript for publication.

Competing interests: All authors have completed the ICMJE uniform disclosure form and declare: MLC and JHV report grants from the William Demant Foundation during the conduct of the study. JHV also received grants from Innovation Fund Denmark and William Demant Foundation, and a joined grant from Hearing aid Industry (Oticon, WSA, GN Hearing), outside the submitted work; no other support for the submitted work; no financial relationships with any organizations that might have an interest in the submitted work in the previous three years; no other relationships or activities that could appear to have influenced the submitted work.

Data sharing: As this study is based on data from the Danish national registers, which belong to the Danish Ministry of Health and Statistics Denmark, the authors are not allowed to share data in raw form.

Ethical approval: This study was approved by the local authorities (record No: 2018-DCRC-0055) and was conducted in accordance with principles of the Declaration of Helsinki. By Danish Law, the study does not require patients' consent and approval from ethical committees, as it is entirely based on data from the Danish national registers. The lead author affirms (MLC) that the manuscript is an honest, accurate, and transparent account of the study being reported; that no important aspects of the study have been omitted; and that any discrepancies from the study as planned (and, if relevant, registered) have been explained.

\section{Dissemination to participants and related patient and public} communities: There are plans to disseminate the results of the research to the general public, mainly through media outreach (eg, press releases by the research institutions of the contributing authors, and plain language publications in popular and social media). Results will also be communicated to relevant clinicians in Denmark.

Provenance and peer review: Not commissioned; externally peer reviewed.

This is an Open Access article distributed in accordance with the Creative Commons Attribution Non Commercial (CC BY-NC 4.0) license, which permits others to distribute, remix, adapt, build upon this work non-commercially, and license their derivative works on different terms, provided the original work is properly cited and the use is noncommercial. See: http://creativecommons.org/licenses/by-nc/4.0/.

1 Livingston G, Huntley J, Sommerlad A, et al. Dementia prevention, intervention, and care: 2020 report of the Lancet Commission. Lancet 2020;396:413-46. doi:10.1016/S0140-6736(20)30367-6

2 Prince M, Comas-Herrera A, Knapp M, Guerchet M, Karagiannidou M. World Alzheimer Report 2016 - Improving healthcare for people living with dementia. London: 2016. https://www.alz.co.uk/research/ world-report-2016

3 Killin LOJ, Starr JM, Shiue IJ, Russ TC. Environmental risk factors for dementia: a systematic review. BMC Geriatr 2016;16:175. doi:10.1186/s12877-016-0342-y

4 Hänninen O, Knol AB, Jantunen M, et al, EBoDE Working Group. Environmental burden of disease in Europe: assessing nine risk factors in six countries. Environ Health Perspect 2014;122:439-46. doi:10.1289/ehp.1206154
5 European Environment Agency. Environmental noise in Europe 2020. Luxembourg: 2020. https://www.eea.europa.eu/publications/ environmental-noise-in-europe.

6 World Health Organization. Environmental noise guidelines for the European Region. 2018. https://www.euro.who.int/en/health-topics/ environment-and-health/noise/publications/2018/environmentalnoise-guidelines-for-the-european-region-2018.

7 Münzel T, Sørensen M, Gori T, et al. Environmental stressors and cardio-metabolic disease: part l-epidemiologic evidence supporting a role for noise and air pollution and effects of mitigation strategies. Eur Heart/ 2017;38:550-6. doi:10.1093/eurheartj/ehw269

8 Münzel T, Schmidt FP, Steven S, Herzog I, Daiber A, Sørensen M. Environmental Noise and the Cardiovascular System. J Am Coll Cardiol 2018;71:688-97. doi:10.1016/j.jacc.2017.12.015

9 Babisch W. The noise/stress concept, risk assessment and research needs. Noise Health 2002:4:1-11.

10 Selander J, Bluhm G, Theorell T, et al, HYENA Consortium. Saliva cortisol and exposure to aircraft noise in six European countries. Environ Health Perspect 2009;117:1713-7. doi:10.1289/ ehp.0900933

11 Basner M, McGuire S. WHO environmental noise guidelines for the european region: A systematic review on environmental noise and effects on sleep. Int J Environ Res Public Health 2018;15:519. doi:10.3390/ijerph15030519

12 Herzog J, Schmidt FP, Hahad O, et al. Acute exposure to nocturnal train noise induces endothelial dysfunction and prothromboinflammatory changes of the plasma proteome in healthy subjects. Basic Res Cardiol 2019;114:46. doi:10.1007/s00395019-0753-y

13 Kröller-Schön S, Daiber A, Steven S, et al. Crucial role for Nox2 and sleep deprivation in aircraft noise-induced vascular and cerebral oxidative stress, inflammation, and gene regulation. Eur Heart /2018:39:3528-39. doi:10.1093/eurheartj/ehy333

14 Münzel T, Daiber A, Steven S, et al. Effects of noise on vascular function, oxidative stress, and inflammation: mechanistic insight from studies in mice. Eur Heart J 2017;38:2838-49. doi:10.1093/ eurheartj/ehx081

15 Schmidt FP, Basner M, Kröger G, et al. Effect of nighttime aircraft noise exposure on endothelial function and stress hormone release in healthy adults. Eur Heart / 2013;34:3508-14a. doi:10.1093/ eurheartj/eht269.

16 Lim ASP, Kowgier M, Yu L, Buchman AS, Bennett DA. Sleep fragmentation and the risk of incident Alzheimer's disease and cognitive decline in older persons. Sleep 2013;36:1027-32. doi:10.5665/sleep.2802

17 Irwin MR, Vitiello MV. Implications of sleep disturbance and inflammation for Alzheimer's disease dementia. Lancet Neurol 2019;18:296-306. doi:10.1016/S1474-4422(18)30450-2

18 Minakawa EN, Wada K, Nagai Y. Sleep disturbance as a potential modifiable risk factor for Alzheimer's disease. Int J Mol Sci 2019;20:1-15. doi:10.3390/ijms20040803

19 Tarantini S, Tran CHT, Gordon GR, Ungvari Z, Csiszar A. Impaired neurovascular coupling in aging and Alzheimer's disease: Contribution of astrocyte dysfunction and endothelial impairment to cognitive decline. Exp Gerontol 2017;94:52-8. doi:10.1016/j. exger.2016.11.004

20 Yamazaki Y, Kanekiyo T. Blood-Brain Barrier Dysfunction and the Pathogenesis of Alzheimer's Disease. Int J Mol Sci 2017;18:1-19. doi:10.3390/ijms18091965.

21 Chen Z, Zhong C. Oxidative stress in Alzheimer's disease. Neurosci Bull 2014;30:271-81. doi:10.1007/s12264-013-1423-y

22 Andersson J, Oudin A, Sundström A, Forsberg B, Adolfsson R, Nordin M. Road traffic noise, air pollution, and risk of dementia - results from the Betula project. Environ Res 2018;166:334-9. doi:10.1016/j. envres.2018.06.008

23 Carey IM, Anderson HR, Atkinson RW, et al. Are noise and air pollution related to the incidence of dementia? A cohort study in London, England. BMJ Open 2018;8:e022404. doi:10.1136/ bmjopen-2018-022404

24 Yuchi W, Sbihi H, Davies H, Tamburic L, Brauer M. Road proximity, air pollution, noise, green space and neurologic disease incidence: a population-based cohort study. Environ Health 2020;19:8. doi:10.1186/s12940-020-0565-4

25 Hegewald J, Schubert M, Freiberg A, et al. Traffic Noise and Mental Health: A Systematic Review and Meta-Analysis. Int J Environ Res Public Health 2020:17:1-26. doi:10.3390/iierph17176175

26 Fuks KB, Wigmann C, Altug H, Schikowski T. Road traffic noise at the residence, annoyance, and cognitive function in elderly women. Int J Environ Res Public Health 2019;16:1-12. doi:10.3390/ ijerph16101790

27 Tzivian L, Dlugaj M, Winkler A, et al, Heinz Nixdorf Recall study Investigative Group. Long-Term Air Pollution and Traffic Noise Exposures and Mild Cognitive Impairment in Older Adults: A CrossSectional Analysis of the Heinz Nixdorf Recall Study. Environ Health Perspect 2016;124:1361-8. doi:10.1289/ehp.1509824 
28 Yu Y, Mayeda ER, Paul KC, et al. Traffic-related noise exposure and late-life dementia and cognitive impairment in MexicanAmericans. Epidemiology 2020;31:771-8. doi:10.1097/ EDE.0000000000001249

29 Schmidt M, Pedersen L, Sørensen HT. The Danish Civil Registration System as a tool in epidemiology. Eur J Epidemiol 2014;29:541-9. doi:10.1007/s10654-014-9930-3

30 Pedersen CB. The Danish civil registration system. Scand J Public Health 2011.39(Suppl):22-5. doi:10.1177/1403494810387965

31 Phung TKT, Waltoft BL, Kessing LV, Mortensen PB, Waldemar G. Time trend in diagnosing dementia in secondary care. Dement Geriatr Cogn Disord 2010;29:146-53. doi:10.1159/000269933

32 Mors O, Perto GP, Mortensen PB. The Danish psychiatric central research register. Scand J Public Health 2011;39(Suppl):54-7. doi:10.1177/1403494810395825.

33 Lynge E, Sandegaard JL, Rebolj M. The Danish national patient register. Scand J Public Health 2011;39(Suppl):30-3. doi: $10.1177 / 1403494811401482$

34 Kildemoes HW, Sørensen HT, Hallas J. The Danish national prescription registry. Scand J Public Health 2011;39(Suppl):38-41. doi:10.1177/1403494810394717

35 Basner M, Babisch W, Davis A, et al. Auditory and non-auditory effects of noise on health. Lancet 2014;383:1325-32. doi:10.1016/ S0140-6736(13)61613-X

36 Bendtsen $\mathrm{H}$. The Nordic prediction method for road traffic noise. Sci Total Environ 1999:235:331-8. doi:10.1016/S0048 9697(99)00216-8

37 Plovsing B, Kragh J. Nord2000. Comprehensive Outdoor Sound Propagation Model. Part 1: Propagation in an Atmosphere without Significant Refraction. Hørsholm: 2006.https://forcetechnology. com/-/media/force-technology-media/pdf-files/projects/nord2000/ nord2000-comprehensive-outdoor-sound-propagation-model---part2-propagation-in-an-atmosphere-with-re.pdf.

38 Ström T. Road traffic noise - an estimate of prediction uncertainty. [In Swedish]. Borås: 1997.

39 Jensen SS, Plejdrup MS, Hillig K. GIS-based National Road and Traffic Database 1960-2020 . Aarhus: 2019.https://dce2.au.dk/pub/ TR151.pdf

40 Thacher JD, Poulsen AH, Raaschou-Nielsen O, et al. High-resolution assessment of road traffic noise exposure in Denmark. Environ Res 2020;182:109051. doi:10.1016/j.envres.2019.109051

41 Levin G, losub C-I, Jepsen MR. BASEMAP02: Technical documentation of a model for elaboration of a land-use and land-cover map for Denmark. 2017. https://dce2.au.dk/pub/TR95.pdf

42 Khan J, Kakosimos K, Raaschou-Nielsen O, et al. Development and performance evaluation of new AirGIS - A GIS based air pollution and human exposure modelling system. Atmos Environ 2019;198:10221. doi:10.1016/j.atmosenv.2018.10.036

43 Danish Health Data Authority. Udvalgte kroniske sygdomme og svære psykiske lidelser. https://www.esundhed.dk/Registre/Register-forudvalgte-kroniske-sygdomme\#tabpanel528A73EA3F03411FA0230 64D0D4AF106

44 Phung TKT, Andersen BB, Høgh P, Kessing LV, Mortensen PB, Waldemar G. Validity of dementia diagnoses in the Danish hospital registers. Dement Geriatr Cogn Disord 2007;24:220-8. doi:10.1159/000107084

45 van Oers JAM, Bongers IMB, van de Goor LAM, Garretsen HFL. Alcohol consumption, alcohol-related problems, problem drinking, and socioeconomic status. Alcohol Alcohol 1999;34:78-88. doi:10.1093/alcalc/34.1.78

46 Roswall N, Christensen JS, Bidstrup PE, et al. Associations between residential traffic noise exposure and smoking habits and alcohol consumption-A population-based study. Environ Pollut 2018;236:983-91. doi:10.1016/j.envpol.2017.10.093

47 Foraster M, Eze IC, Vienneau D, et al. Long-term transportation noise annoyance is associated with subsequent lower levels of physical activity. Environ Int 2016;91:341-9. doi:10.1016/j. envint.2016.03.011

48 Phung TKT, Andersen BB, Kessing LV, Mortensen PB, Waldemar $G$. Diagnostic evaluation of dementia in the secondary health care sector. Dement Geriatr Cogn Disord 2009;27:534-42. doi:10.1159/000223664

49 Pickett J, Bird C, Ballard C, et al. A roadmap to advance dementia research in prevention, diagnosis, intervention, and care by 2025. Int J Geriatr Psychiatry 2018;33:900-6. doi:10.1002/gps.4868

50 Vienneau D, Héritier H, Foraster M, et al, SNC study group. Façades, floors and maps - Influence of exposure measurement error on the association between transportation noise and myocardial infarction. Environ Int 2019:123:399-406. doi:10.1016/i.envint.2018.12.015

51 Chen H, Kwong JC, Copes R, et al. Exposure to ambient air pollution and the incidence of dementia: A population-based cohort study. Environ Int 2017;108:271-7. doi:10.1016/j.envint.2017.08.020

52 Peters R, Ee N, Peters J, Booth A, Mudway I, Anstey KJ. Air Pollution and Dementia: A Systematic Review. J Alzheimers Dis 2019;70(s1):S145-63. doi:10.3233/JAD-180631

53 Sørensen M. Pershagen G. Transportation noise linked to cardiovascular disease independent from air pollution. Eur Heart / 2019;40:604-6. doi:10.1093/eurheartj/ehy768

54 Bodin T, Björk J, Ardö J, Albin M. Annoyance, sleep and concentration problems due to combined traffic noise and the benefit of quiet side. Int J Environ Res Public Health 2015;12:1612-28. doi:10.3390/ ijerph120201612

55 Münzel T, Kröller-Schön S, Oelze M, et al. Adverse cardiovascular effects of traffic noise with a focus on nighttime noise and the new WHO noise guidelines. Annu Rev Public Health 2020;41:309-28. doi:10.1146/annurev-publhealth-081519-062400

56 Li W, Su D, Zhai Q, et al. Proteomes analysis reveals the involvement of autophagy in AD-like neuropathology induced by noise exposure and ApoE4. Environ Res 2019;176:108537. doi:10.1016/j. envres.2019.108537

57 Cui B, Su D, Li W, et al. Effects of chronic noise exposure on the microbiome-gut-brain axis in senescence-accelerated prone mice: implications for Alzheimer's disease. / Neuroinflammation 2018;15:190. doi:10.1186/s12974-018-1223-4

58 Jafari Z, Kolb BE, Mohajerani MH. Noise exposure accelerates the risk of cognitive impairment and Alzheimer's disease: Adulthood, gestational, and prenatal mechanistic evidence from animal studies. Neurosci Biobehav Rev 2020;117:110-28. doi:10.1016/j. neubiorev.2019.04.001

59 Su D, Li W, Chi H, et al. Transcriptome analysis of the hippocampus in environmental noise-exposed SAMP8 mice reveals regulatory pathways associated with Alzheimer's disease neuropathology. Environ Health Prev Med 2020;25:3. doi:10.1186/s12199-0190840-6

60 Gomperts SN. Lewy Body Dementias: Dementia With Lewy Bodies and Parkinson Disease Dementia. Continuum (Minneap Minn) 2016;22(2 Dementia):435-63. doi:10.1212/ CON.0000000000000309

61 Duong S, Patel T, Chang F. Dementia: What pharmacists need to know. Can Pharm I (Ott) 2017:150:118-29. doi:10.1177/1715163517690745

62 Sørensen M, Poulsen AH, Hvidtfeldt UA, et al. Transportation noise and risk of stroke: a nationwide prospective cohort study covering Denmark. Int J Epidemiol 2021;03:1-10. doi:10.1093/ije/ dyab024

63 Pyko A, Andersson N, Eriksson C, et al. Long-term transportation noise exposure and incidence of ischaemic heart disease and stroke: a cohort study. Occup Environ Med 2019;76:201-7. doi:10.1136/ oemed-2018-105333

64 Seidler AL, Hegewald J, Schubert M, et al. The effect of aircraft, road, and railway traffic noise on stroke - results of a case-control study based on secondary data. Noise Health 2018;20:152-61.

65 European Commision - Science for Environment Policy. FUTURE BRIEF: Noise abatement approaches. Luxembourg: 2017. doi: $10.2779 / 016648$

66 Thacher JD, Hvidtfeldt UA, Poulsen AH, et al. Long-term residential road traffic noise and mortality in a Danish cohort. Environ Res 2020;187:109633. doi:10.1016/j.envres.2020.109633

67 Babisch W, Pershagen G, Selander J, et al. Noise annoyance--a modifier of the association between noise level and cardiovascular health?Sci Total Environ 2013;452-453:50-7. doi:10.1016/j. scitotenv.2013.02.034

68 Foraster M, Künzli N, Aguilera I, et al. High blood pressure and long-term exposure to indoor noise and air pollution from road traffic. Environ Health Perspect 2014;122:1193-200. doi:10.1289/ ehp.1307156

69 Eze IC, Foraster M, Schaffner E, et al. Long-term exposure to transportation noise and air pollution in relation to incident diabetes in the SAPALDIA study. Int J Epidemiol 2017;46:1115-25. doi:10.1093/ije/dyx020

Supplementary information: additional tables 1-8 and figures 1-6 